\title{
Search for magnetic monopoles with Belle II experiment
}

\author{
Neverov Dmitrii* \\ Nagoya University \\ E-mail: neverov@hepl.phys.nagoya-u.ac.jp, D.I.Neverov@gmail.com
}

\begin{abstract}
A magnetic monopole is a stable particle carrying magnetic charge, it is suggested by the symmetry of Maxwell's equations and appears in e.g. Grand Unification Theories. Originally it was proposed by Dirac in 1931 as a way to quantize electric charge: $e_{0} g_{0}=n \hbar c / 2$, thus giving minimal Dirac charge $g_{D}=68.5 e$. Modern experiments focus on high ionization of a Dirac charge, while lower charge monopoles predicted by other theories are easy to be missed without a dedicated tracking and trigger setup. In this work, an overview of the search for magnetic monopoles with Belle II detector is presented.
\end{abstract}

The 4th KMI International Symposium (KMI2019)

18-20, February 2019

Nagoya, Japan

${ }^{*}$ Speaker. 


\section{Introduction}

A magnetic monopole is a stable particle carrying magnetic charge, it is suggested by the symmetry of Maxwell's equations and appears in e.g. Grand Unification Theories [2][3]. In a formal way it was originally proposed by Dirac in 1931 [1] as a way to explain electric charge quantization. It was shown that it is possible to construct a pole in the magnetic field flux as an end of an infinitely thin solenoid called Dirac String. If one then considers the change in the wave function phase of an electrically charged particle around a closed curve near the magnetic pole, one can come up with the following condition on the particle electric charge $e_{0}$ and the strength of that magnetic pole $g_{0}: e_{0} g_{0}=n \hbar c / 2$

If the minimal (free) electric charge is that of an electron, the minimal magnetic charge is $g_{D} \approx 68.5 e$, called Dirac charge.

Same relation can be obtained by considering the angular momentum of separated electric and magnetic charges as was shown by Saha [4].

Magnetic monopoles have been searched for in cosmic rays, in Earth crust and in particle collision experiments, and none have been found except a couple of non-conclusive events [7][8]. Modern experiments focus on the high ionization of a Dirac charge e.g. searches by MoEDAL [10], sensitive to magnetic charges $g>68.5 e$, and by ATLAS [9] at the LHC which is sensitive to charges $34 e<g<137 e$, while the latest searches for lower charge monopoles have been done by TASSO [11] in 1988 in the region of $10 e<g<70 e$ and CLEO [12] in 1987 with magnetic charges $2 e<g<10 e$.

Note that there have been no direct searches for monopoles with the magnetic charge $g=1 e$, and even if they were to be produced in particle collision experiments, as some suggest [5][6][18], they would be easy to miss without a dedicated tracking and trigger setup.

\section{Belle II experiment}

The Belle II experiment is a substantial upgrade of the Belle detector and will operate at the SuperKEKB energy-asymmetric $e^{+} e^{-}$collider. The accelerator has successfully completed the commissioning phases in 2016 and 2018 and has started the physics data taking phase in April 2019. The design luminosity of SuperKEKB is $8 \times 10^{35} \mathrm{~cm}^{-2} \mathrm{~s}^{-1}$ and the Belle II experiment aims to record $50 \mathrm{ab}^{-1}$ of data, a factor of 50 more than the Belle experiment. The main physics program is described in great detail in [13].

The trigger setup during the early stages of data taking is quite loose so that if monopole events occur they will be saved and be available for the offline analysis. At the same time the Belle II software framework basf2 [14] is being actively developed which allows for the said analysis to happen.

\section{Signature}

Since the magnetic charge is a conserved quantity, magnetic monopoles would be produced as a pair of north $(g<0)$ and south $(g>0)$ monopoles in an $e^{+} e^{-}$collision (Fig. 1), provided the 
energy is above the mass threshold.

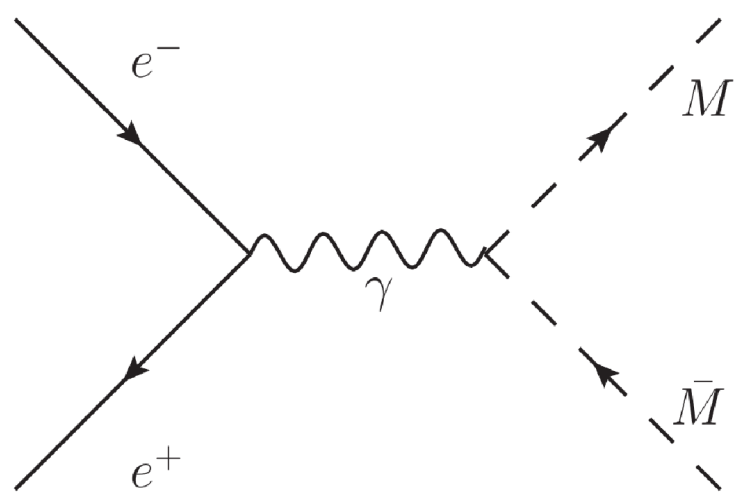

Figure 1: A Feynman diagram of monopole antimonopole pair production

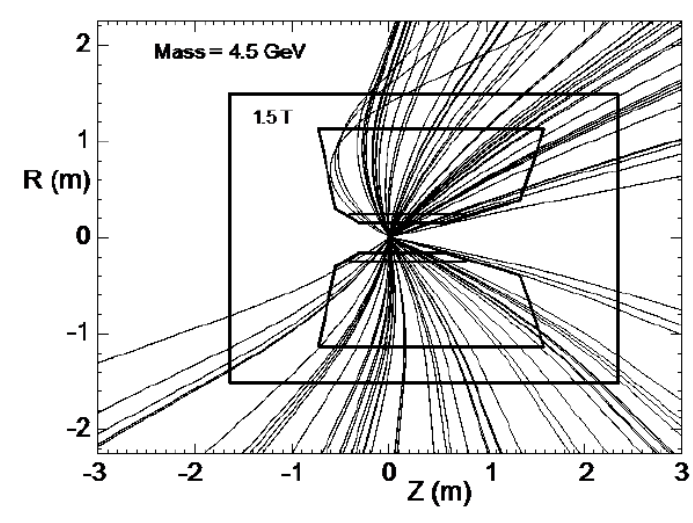

Figure 2: Possible topologies of monopole stereo tracks [18]

The behavior of a monopole in the Belle II magnetic field would be similar to that of an electrically charged particle in an electric field. Tracks left by a monopole would be non-helical straight in the $(R, \phi)$ plane perpendicular to the magnetic field, and curved along the magnetic lines in the $(R, z)$ plane as shown in Fig. 2.

Compared to the linear $z(s)=z_{0}+s \tan \lambda$ dependence for electrically charged particles, magnetic tracks have an extra quadratic term coming from the acceleration in the magnetic field

$$
z(t)=z_{0}+t \frac{p_{\| c}}{E}+t^{2} \frac{g B c}{2 E}
$$

which is a semi-classical approximation of a catenary in the relativistic solution.

The non-helical tracks are a clear signature to be looked for in the Belle II data and the offline analysis can be done following CLEO procedure [12], by performing linear and quadratic fits to the monopole track candidates and looking (Fig. 3) at the $\chi^{2}$ discriminator

$$
F=\frac{\chi_{L}^{2}-\chi_{Q}^{2}}{\chi_{L}^{2} /(N-3)}
$$

Simulation with GEANT4 [15], as described in the following section, shows that pairs of monopoles produced within ranges $m<5 \mathrm{GeV} / \mathrm{c}^{2}$ and $g<5 e$ traverse enough layers of Belle II Central Drift Chamber (CDC) and reach Electromagnetic calorimeter (ECL) giving the hits to work with. There are also hits left in the Vertex Detector (VXD) and K-long and muon calorimeter (KLM) but they are not considered at the current stage.

\section{Simulation}

The GEANT4 simulation of monopole pair production is implemented with the Belle II geometry and magnetic field within the bas 2 framework, and monopoles are propagated with the Lorentz force for the magnetic charge

$$
F=g(B-\beta \times E)
$$




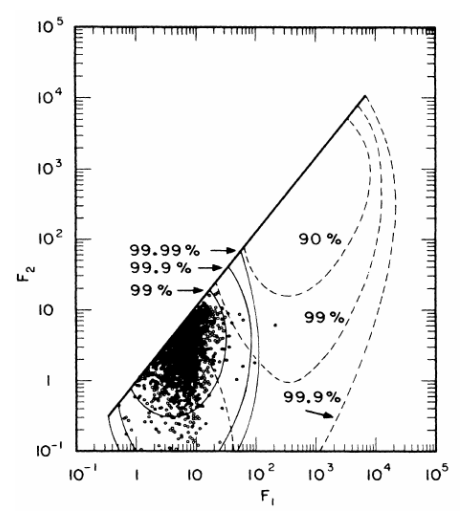

Figure 3: Discriminator performance on track pair at CLEO

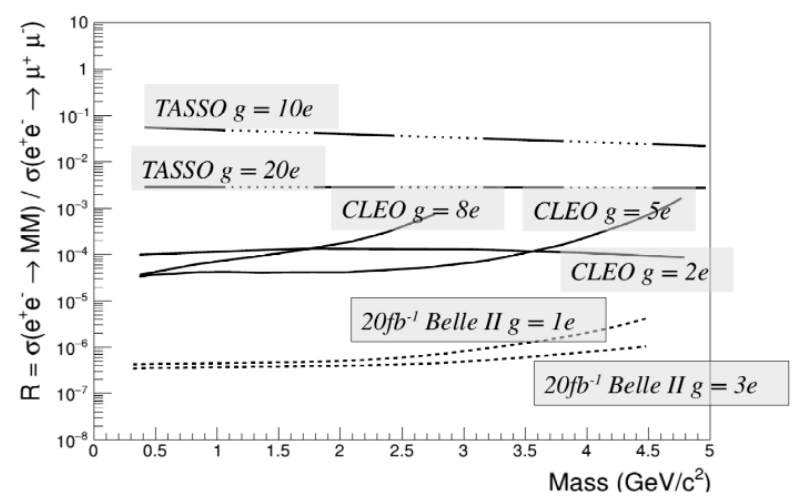

Figure 4: Projected sensitivity to production of magnetic monopoles in Belle II

The ionisation loss coded in the simulation is similar to Bethe-Bloch formula for electric charges but with the charge strength changed to $g \beta$, thus not having the $1 / \beta^{2}$ dependence observed for electric charges at small speeds.

The formula for high magnetic charges was shown by Ahlen [17] and can be applied to lower charges by dropping some of the corrections.

$$
-\frac{d E}{d x}=\frac{4 \pi N_{A} Z g^{2} \alpha^{2}(\hbar c)^{2}}{A m_{e} c^{2}}\left[\ln \left(\frac{2 \gamma^{2} m_{e} \beta^{2} c^{2}}{I}\right)-\frac{1}{2}\right]
$$

L1 triggers that are most sensitive to the monopole pair events were found to be ECL triggers for clusters that are back to back in $(R, \phi)$ plane.

\section{Tracking}

The tracking in CDC of Belle II can be viewed as done in several stages:

- axial track finding

- stereo track finding

- track fitting

Following this structure a modification of conventional tracking was developed. First, a dedicated track finder suitable to look for straight tracks in the $(R, \phi)$ plane from axial wire hits of CDC takes all hits compatible with a line coming from the Interaction Point (IP) to an ECL cluster with energy higher than given.

The obtained axial track candidate is used to reconstruct $z$ coordinate positions of hits in the stereo layers of CDC, which are then given to a quadtree based Hough algorithm that searches for a hit set with a quadratic $z(s)$ dependence.

Monopole track candidate is the set of axial and stereo hits found by previous two steps and in order to obtain monopole parameters, a fit will be performed with the GenFit [16] toolkit with an implementation yet to be done. 


\section{Conclusion}

Because of the opportunity in the parameter space and a clear experimental signature this is a promising search to be done using the early data of Belle II. With the data collected during the commissioning run and the beginning of physics run, it is possible to perform first ever direct search for magnetic monopoles of charge $g=1 e$ and revisit parameter space region of $g<5 e$ and $m<5 \mathrm{GeV} / c$ with better sensitivity of Belle II (Fig. 4).

\section{References}

[1] P.A.M. Dirac, Quantised singularities in the electromagnetic field, Proc. Roy. Soc. Lond. A133, 60 (1931)

[2] G.'t Hooft, Magnetic Monopoles in Unified Gauge Theories, Nucl. Phys. B79, 276 (1974)

[3] A.M. Polyakov, Particle Spectrum in the Quantum Field Theory, JETP Lett. 20, 194 (1974) [Pisma Zh. Eksp. Teor. Fiz. 20, 430 (1974)]

[4] M. N. Saha, Note on Dirac's Theory of Magnetic Poles, Phys. Rev. 75, 1968 - Published 15 June 1949

[5] D. Fryberger, A Model For The Structure Of Point - Like Fermions, Qualitative Features And Physical Description, Found.Phys. 13 (1983) 1059

[6] D. Fryberger, A Semiclassical Monopole Configuration for Electromagnetism, Hadronic J 4 (1981) 1844

[7] B. Cabrera, First Results from a Superconductive Detector for Moving Magnetic Monopoles, Phys.Rev.Lett. 48 (1982) 1378-1380

[8] A.D. Caplin, M. Hardiman, M. Koratzinos, J.C. Schouten, Observation of an Unexplained Event From a Magnetic Monopole Detector, Nature 321 (1986) 402-406

[9] ATLAS collaboration, Search for magnetic monopoles and stable particles with high electric charges in $8 \mathrm{TeV}$ pp collisions with the ATLAS detector, Phys.Rev. D93 (2016) no.5, 052009

[10] MoEDAL collaboration, Search for Magnetic Monopoles with the MoEDAL Forward Trapping Detector in $13 \mathrm{TeV}$ Proton-Proton Collisions at the LHC, Phys.Rev.Lett. 118 (2017) no.6, 061801

[11] TASSO Collaboration, W. Braunschweig, R. Gerhards, et al., A search for particles with magnetic charge produced $e^{+} e^{-}$annihilations at $\sqrt{s}=35 \mathrm{GeV}$, Z. Phys. C - Particles and Fields (1988) 38: 543.

[12] CLEO Collaboration, T. Gentile et al., Search for magnetically charged particles produced in $e^{+} e^{-}$ annihilations at $\sqrt{s}=10.6$ GeV, Phys. Rev. D 35, 1081(R) (1987)

[13] E. Kou, P. Urquijo et al., The Belle II Physics Book, To be published in PTEP [hep-ex/1808.10567]

[14] T. Kuhr, C. Pulvermacher, M. Ritter, et al., The Belle II Core Software, Comput. Softw. Big Sci. 3 (2019) no.1 [comp-ph/1809.04299]

[15] GEANT4 collaboration, Recent developments in Geant4, Nucl.Instrum.Meth. A835 (2016) 186-225, https://geant4.web.cern.ch/

[16] https://github.com/GenFit/GenFit

[17] S. P. Ahlen, Theoretical and experimental aspects of the energy loss of relativistic heavily ionizing particles, Rev. Mod. Phys. 52, 121 (1980)

[18] M. K. Sullivan, D. Fryberger, Magnetic Charge Search for the BELLE II Detector [hep-ex/1707.05295] 